\title{
Perturbative Treatment of the Evolution Operator Associated with Raman Couplings
}

\author{
Benedetto MILITELLO ${ }^{\dagger}$, Paolo ANIELLO ${ }^{\ddagger}$ and Antonino MESSINA ${ }^{\dagger}$ \\ ${ }^{\dagger}$ INFM, MIUR and Dipartimento di Scienze Fisiche ed Astronomiche dell'Università di Palermo, \\ Via Archiraf $i, 36$ - 90123 Palermo, Italy \\ E-mail:bdmilite@fisica.unipa.it,messina@fisica.unipa.it \\ $\ddagger$ Dipartimento di Scienze Fisiche dell'Università di Napoli 'Federico II' and INFN Sezione \\ di Napoli, Complesso Universitario di Monte S. Angelo, Via Cintia - 80126 Napoli, Italy \\ E-mail: paolo.aniello@na.infn.it
}

Received November 02, 2005, in final form April 13, 2006; Published online May 10, 2006

Original article is available at http://www.emis.de/journals/SIGMA/2006/Paper050/

\begin{abstract}
A novel perturbative treatment of the time evolution operator of a quantum system is applied to the model describing a Raman-driven trapped ion in order to obtain a suitable 'effective model'. It is shown that the associated effective Hamiltonian describes the system dynamics up to a certain transformation which may be interpreted as a 'dynamical dressing' of the effective model.
\end{abstract}

Key words: perturbation theory; time-dependent problems; Raman couplings

2000 Mathematics Subject Classification: 81Q15; 81V10; 81V45

\section{Introduction}

Over the last few years more and more attention has been addressed to the analysis of physical nano-systems in order to realize intriguing applications, for instance in the field of quantum computation, and to investigate fundamental aspects of quantum mechanics. One of the most promising physical contexts is that of laser-driven trapped ions (for a review see, for instance $[1,2])$.

An electromagnetic (e.m.) trap is a device which generates a suitable e.m. field confining a charged particle in a finite region of space. In particular, a Paul trap exploits an inhomogeneous and time-dependent e.m. field which forces a charged particle to move approximately as a harmonic oscillator. The equilibrium point coincides with the center of the trap [3]. Therefore, a trapped ion is describable as a compound system made of a three-dimensional quantum harmonic oscillator (representing the motion of the ion center of mass inside the trap) and a few-level system (associated with the internal atomic state, i.e. with the 'relevant electronic levels' of the ion). Through the action of suitable driving laser fields, it is possible to coherently manipulate this system with a high degree of accuracy. In particular, the possibility of inducing couplings between the atomic degrees of freedom and the ion center of mass motion has been experimentally demonstrated in a very wide variety of settings $[1,2,3]$.

In many experimental situations, only two atomic states are effectively involved in the dynamics of a ion trap. In fact, such two states are the only two effectively coupled by the laser fields driving the ion. Such effective couplings, for technical reasons, are usually realized through a third atomic level. Precisely, the two effective atomic levels - say $|1\rangle$ and $|2\rangle$ - are nonresonantly coupled to a third level - the auxiliary level $|3\rangle$ - and the respective two 'amounts of off-resonance' (i.e. the two detunings, that is the differences between the atomic Bohr frequencies 
and the corresponding laser frequencies) are chosen to be equal [4]. The idea behind such a coupling scheme - the 'Raman scheme' - is that direct transitions from (and to) level $|3\rangle$ to (and from) the other two levels are forbidden by the energy conservation, while two-photon processes bringing from (and to) level $|1\rangle$ to (and from) level $|2\rangle$, through the auxiliary level $|3\rangle$, are possible, and this realizes an effective coupling $|1\rangle \rightleftarrows|2\rangle$. We will show that such a reasoning is correct within a certain approximation which will be clarified in the following (see Sections 4 and 5).

In the present paper, we analyze the Raman coupling schemes realized by laser-driven trapped ions deducing, by means of a rigorous perturbative approach, the expressions of the relevant effective couplings. To this aim, we exploit a recently introduced perturbative method based on a suitable decomposition of the time evolution operator associated with a quantum Hamiltonian [5] (for the case of a time-independent Hamiltonian, see also $[6,7,8]$ ). In particular, we investigate the problem of determining the effective Hamiltonian - i.e. the Hamiltonian describing the effective couplings - in the case where two or more Raman coupling schemes are simultaneously active, so providing a rigorous proof of the additivity of the effective couplings.

We will show, moreover, that in the special case of a single Raman coupling our result coincides with the result previously obtained by means of a time-independent perturbative approach [9].

The paper is organized as follows. In Section 2, we describe the general form of the Hamiltonian associated with a trapped ion Raman scheme. In Section 3, we introduce the perturbative method which is the main tool of the paper. We apply this method in Section 4, where we analyze the dynamics of a double $\Lambda$ Raman scheme. Finally, in Section 5, some conclusive remarks are drawn.

\section{The physical system}

The general form of the quantum Hamiltonian of a trapped three-level ion addressed by a set of laser beams coupling the atomic level $|3\rangle$ with the other two levels (with suitable ion-laser detunings) is the following:

$$
H(t)=H_{0}+H_{\mathrm{B}}+H_{\mathrm{R}}(t)
$$

where

$$
\begin{aligned}
& H_{0}=\sum_{l=1,2,3} \hbar \omega_{l} \hat{\sigma}_{l l}, \quad H_{\mathrm{B}}=\hbar \nu \sum_{\alpha=x, y, z} \hat{a}_{\alpha}^{\dagger} \hat{a}_{\alpha}, \\
& H_{\mathrm{R}}(t)=\left(\hbar \hat{\Omega}_{13}(t) \hat{\sigma}_{13}+\text { h.c. }\right)+\left(\hbar \hat{\Omega}_{23}(t) \hat{\sigma}_{23}+\text { h.c. }\right),
\end{aligned}
$$

with $\hat{\sigma}_{l m} \equiv|l\rangle\langle m|, l, m=1,2,3,\{|l\rangle\}_{l=1}^{3}$ being the considered three atomic levels and $\left\{\hbar \omega_{l}\right\}_{l=1}^{3}$ the corresponding energies $\left(\omega_{l} \neq \omega_{r}\right.$, for $\left.l \neq r\right) ;\left\{\hat{a}_{\alpha}: \alpha=x, y, z\right\}$ are the annihilation operators associated with the center of mass harmonic motion along the axes $x, y, z$, namely,

$$
\hat{a}_{x}=\left(\frac{\mu \nu}{2 \hbar}\right)^{1 / 2}\left(\hat{x}+\frac{i}{\mu \nu} \hat{p}_{x}\right), \quad \ldots, \quad \hat{a}_{z}=\left(\frac{\mu \nu}{2 \hbar}\right)^{1 / 2}\left(\hat{z}+\frac{i}{\mu \nu} \hat{p}_{z}\right),
$$

which, of course, satisfy the well known bosonic commutation relations $\left[\hat{a}_{\alpha}, \hat{a}_{\beta}\right]=\left[\hat{a}_{\alpha}^{\dagger}, \hat{a}_{\beta}^{\dagger}\right]=0$, $\left[\hat{a}_{\alpha}, \hat{a}_{\beta}^{\dagger}\right]=\delta_{\alpha \beta}$, for all $\alpha, \beta=x, y, z$. Without loss of generality, the three harmonic oscillator frequencies have been taken to be equal: $\nu_{x}=\nu_{y}=\nu_{z} \equiv \nu>0$; thus, we deal with a spherically symmetric trap.

We have denoted by $t \mapsto \hat{\Omega}_{j 3}(t), j=1,2$, operator-valued functions acting in the bosonic Hilbert space (i.e. the Hilbert space associated with the vibrational degrees of freedom); their specific structure is determined by the specific laser configuration. For instance, for a single 
' $\Lambda$ Raman coupling' - involving two laser beams with complex strengths (proportional to the laser amplitudes and to the atomic dipole operators, and including the laser phases), wave vectors and frequencies $g_{13}, \vec{k}_{13}, \omega_{13}$ and $g_{23}, \vec{k}_{23}, \omega_{23}$, respectively - with the lasers in a 'travelling wave configuration', we have:

$$
\hat{\Omega}_{13}(t)=\hbar g_{13} e^{-i\left(\vec{k}_{13} \cdot \vec{r}-\omega_{13} t\right)}, \quad \hat{\Omega}_{23}(t)=\hbar g_{23} e^{-i\left(\vec{k}_{23} \cdot \vec{r}-\omega_{23} t\right)},
$$

where the laser frequencies are fixed in such a way that the two couplings share the same ion-laser detuning

$$
\Delta \equiv \omega_{3}-\omega_{1}-\omega_{13}=\omega_{3}-\omega_{2}-\omega_{23} \neq 0
$$

in order to allow the typical 'two-photon processes' $|1\rangle \rightleftarrows|2\rangle$ of the Raman scheme. In (4) the vector operator $\vec{r}:=(\hat{x}, \hat{y}, \hat{z})$ is the ion center of mass position operator and its presence in the interaction Hamiltonian $H_{\mathrm{R}}(t)$ is responsible for the interaction between atomic and vibrational degrees of freedom. The link between this operator and the annihilation and creation operators stems from relations (3). With regard to the coefficients $g_{13}$ and $g_{23}$ appearing in the definition of the operators $\hat{\Omega}_{13}(t)$ and $\hat{\Omega}_{23}(t)$ respectively, we recall that they are given by $g_{j 3}:=-\hbar^{-1} \vec{d}_{j 3} \cdot \vec{E}_{j 3}$, where $\vec{E}_{j 3}$ is the complex amplitude (i.e. including information about the initial phase of the field) of the laser field tuned near the $|j\rangle \rightarrow|3\rangle$ Bohr frequency, while $\vec{d}_{j 3}$ is the atomic dipole operator matrix element involving the atomic states $|j\rangle$ and $|3\rangle$.

A relevant feature of the laser configuration specified by relations (4) is that, in this particular case, the Hamiltonian $H(t)$ can be transformed, by passing to a suitable rotating frame (i.e. by switching to an ad hoc interaction picture), into a time-independent Hamiltonian, which can be then treated by means of a time-independent perturbative approach, see [9].

However, more complicated laser configurations are possible and useful for various applications, and, in general, one cannot find a simple rotating frame where the Hamiltonian of the system becomes time-independent. Therefore, it will be convenient to apply a time-dependent perturbative approach.

\section{The time-dependent perturbative approach}

In order to study the class of dynamical problems associated with a quantum Hamiltonian of the form (1), one can fruitfully exploit a time-dependent perturbative method based on a suitable decomposition of the evolution operator [5] which is a generalization of the classical Magnus expansion [10].

Consider a quantum system whose Hamiltonian is made of two components, the unperturbed energy operator $H_{0}$, and a perturbation $H_{\diamond}(\lambda ; t)$, in general time-dependent:

$$
H(\lambda ; t)=H_{0}+H_{\diamond}(\lambda ; t) .
$$

We assume that $\lambda \mapsto H_{\diamond}(\lambda ; t)$ is an analytic function of the (real) perturbative parameter $\lambda$ $\left(H_{\diamond}(0 ; t)=0\right)$.

Introducing the evolution operator generated by the unperturbed component $H_{0}$ - namely, $U_{0}:=e^{-\frac{i}{\hbar} H_{0} t}-$ and the the evolution operator $T(\lambda ; t)$ associated with the interaction picture Hamiltonian $\tilde{H}(\lambda ; t):=U_{0}(t)^{\dagger} H_{\diamond}(\lambda ; t) U_{0}(t)$, it is possible to factorize the total evolution operator $U(\lambda ; t)$ of the system as

$$
U(\lambda ; t)=U_{0}(t) T(\lambda ; t)
$$


We can now consider the following exact decomposition of the interaction picture evolution operator $T(\lambda ; t)$ as a product of unitary operators:

$$
T(\lambda ; t)=\exp (-i Z(\lambda ; t)) \exp \left(-i \int_{0}^{t} C(\lambda ; \mathfrak{t}) \mathrm{d} \mathfrak{t}\right) \exp (i Z(\lambda)),
$$

where $Z(\lambda ; t), C(\lambda ; t), Z(\lambda)$ are selfadjoint operators and

$$
Z(\lambda) \equiv Z(\lambda ; 0), \quad Z(0 ; t)=C(0 ; t)=0, \quad \forall t .
$$

It is worth noting that no time-ordering operator is present in (6). Moreover, observe that in the special case where $Z(\lambda ; t)=0, \forall t$, we have the Magnus expansion, provided that $C(\lambda ; t)$ is regarded as the time derivative of the Magnus unitary operator generator, see [10]; but, as we will see below, decomposition (6) is actually a generalization of the Magnus expansion.

\subsection{Imposing a gauge condition}

It can be shown that the operators $C(\lambda ; t)$ and $Z(\lambda ; t)$ are not uniquely determined in decomposition (6): in fact, there are infinite possible solutions, namely, solutions compatible with the general form of such decomposition. A unique solution can be singled out by imposing an additional 'gauge condition'. A typical example is the case where the interaction picture Hamiltonian $\tilde{H}(\lambda ; t)$ is a almost periodic ${ }^{1}$ operator-valued function of time, in particular, an operator-valued trigonometric polynomial with respect to the time variable. In this case, a remarkable gauge is fixed by the following tern of conditions (see [5]):

1) $C(\lambda ; t)=C(\lambda ; 0) \equiv C(\lambda), \forall t$;

2) the function $t \mapsto Z(\lambda ; t)$ satisfies:

$$
\lim _{t \rightarrow \infty} t^{-1} Z(\lambda ; t)=0
$$

3) the mean value of the function $t \mapsto Z(\lambda ; t)$ is zero, namely:

$$
\lim _{\tau \rightarrow \infty} \frac{1}{\tau} \int_{0}^{\tau} Z(\lambda ; t) \mathrm{d} t=0
$$

As it will be seen in Section 4, this is precisely the case occurring in our applications, due to the fact that the operators $\hat{\Omega}_{13}(t), \hat{\Omega}_{23}(t)$ are indeed trigonometric polynomials:

$$
\hat{\Omega}_{j 3}(t)=\sum_{\kappa=1}^{\bar{\kappa}_{j}} \hat{\Omega}_{j 3}^{\kappa} e^{i \omega_{j}^{\kappa} t}, \quad \bar{\kappa}_{j} \in \mathbb{N}, \quad\left\{\omega_{j}^{1}, \ldots, \omega_{j}^{\bar{\kappa}_{j}}\right\} \subset \mathbb{R}, \quad j=1,2,
$$

where $\left\{\hat{\Omega}_{j}^{1}, \ldots, \hat{\Omega}_{j}^{\bar{\kappa}_{j}}\right\}_{j=1}^{2}$ are operators acting on the bosonic degrees of freedom; see, for instance, relations (4).

\subsection{Determination of $C(\lambda ; t)$ and $Z(\lambda ; t)$ up to a gauge condition}

Using the formula - reported, for instance, in [12] - for the derivative of the exponential of an operator-valued function $t \mapsto F(t)$, i.e.

$$
\frac{\mathrm{d}}{\mathrm{d} t} e^{F}=e^{F} \int_{0}^{1}\left(e^{-s F} \dot{F} e^{s F}\right) \mathrm{d} s=\int_{0}^{1}\left(e^{s F} \dot{F} e^{-s F}\right) \mathrm{d} s e^{F},
$$

\footnotetext{
${ }^{1} \mathrm{~A}$ standard reference on almost periodic functions is [11].
} 
we can write the Schrödinger equation for the interaction picture evolution operator as

$$
\begin{aligned}
\tilde{H}(\lambda ; t) T(\lambda ; t)= & i \hbar \dot{T}(\lambda ; t)=\hbar e^{-i Z(\lambda ; t)} \int_{0}^{1}\left(e^{i s Z(\lambda ; t)} \dot{Z}(\lambda ; t) e^{-i s Z(\lambda ; t)}\right) \mathrm{d} s e^{-i \int_{0}^{t} C(\lambda ; \mathfrak{t}) \mathrm{d} \mathfrak{t}} e^{i Z(\lambda)} \\
& +\hbar e^{-i Z(\lambda ; t)} \int_{0}^{1}\left(e^{-i s \int_{0}^{t} C(\lambda ; \mathfrak{t}) \mathrm{d} t} C(\lambda ; t) e^{i s \int_{0}^{t} C(\lambda ; \mathfrak{t}) \mathrm{d} \mathfrak{t}}\right) \mathrm{d} s e^{-i \int_{0}^{t} C(\lambda ; \mathfrak{t}) \mathrm{d} \mathfrak{t}} e^{i Z(\lambda)}
\end{aligned}
$$

Applying to each member of equation (7) the operator $e^{i Z(\lambda ; t)}$ on the left and the operator $e^{-i Z(\lambda)} e^{i \int_{0}^{t} C(\lambda ; \mathfrak{t}) \mathrm{dt}}$ on the right, we get the following equation relating the operators $C(\lambda ; t)$ and $Z(\lambda ; t)$ with the interaction picture Hamiltonian:

$$
\operatorname{Ad}_{\exp (i Z(\lambda ; t))} \tilde{H}(\lambda ; t)=\hbar \int_{0}^{1}\left(\operatorname{Ad}_{\exp (i s Z(\lambda ; t))} \dot{Z}(\lambda ; t)+\operatorname{Ad}_{\exp \left(-i s \int_{0}^{t} C(\lambda ; \mathfrak{t}) \mathrm{d} \mathfrak{t}\right)} C(\lambda ; t)\right) \mathrm{d} s .
$$

We recall that, given linear operators $\mathfrak{X}$ (invertible) and $Y, \operatorname{Ad}_{\mathfrak{X}} Y:=\mathfrak{X} Y \mathfrak{X}^{-1}$. If the operator $\mathfrak{X}$ is of the form $\mathfrak{X}=e^{X}$, we can use the well known relation

$$
\operatorname{Ad}_{\exp (X)} Y=\exp \left(\operatorname{ad}_{X}\right) Y=\sum_{m=0}^{\infty} \frac{1}{m !} \operatorname{ad}_{X}^{m} Y
$$

with $\operatorname{ad}_{X}^{m}$ denoting the $m$-th power $\left(\operatorname{ad}_{X}^{0} \equiv \mathrm{Id}\right)$ of the adjoint super-operator $\operatorname{ad}_{X}$ defined by $\operatorname{ad}_{X} Y:=[X, Y]$. Applying formula (9) to equation (8), and performing the integrals, we obtain:

$$
\begin{aligned}
\sum_{m=0}^{\infty} \frac{i^{m}}{m !} \operatorname{ad}_{\hat{Z}(\lambda ; t)}^{m} \tilde{H}(\lambda ; t)= & \hbar \sum_{m=0}^{\infty} \frac{i^{m}}{(m+1) !} \operatorname{ad}_{\hat{Z}(\lambda ; t)}^{m} \dot{Z}(\lambda ; t) \\
& +\hbar \sum_{m=0}^{\infty} \frac{(-i)^{m}}{(m+1) !} \operatorname{ad}_{\int_{0}^{t} C(\lambda ; \mathfrak{t}) \mathrm{d} \mathfrak{t}}^{m} C(\lambda ; t) .
\end{aligned}
$$

Next, using the Taylor expansions of the operator-valued functions $\lambda \mapsto \tilde{H}(\lambda ; t), \lambda \mapsto Z(\lambda ; t)$ and $\lambda \mapsto C(\lambda ; t)$ (recall that $\tilde{H}(0 ; t)=Z(0 ; t)=C(0 ; t)=0)$, i.e.

$$
\tilde{H}(\lambda ; t)=\sum_{n=1}^{\infty} \lambda^{n} \tilde{H}_{n}(t), \quad Z(\lambda ; t)=\sum_{n=1}^{\infty} \lambda^{n} Z_{n}(t), \quad C(\lambda ; t)=\sum_{n=1}^{\infty} \lambda^{n} C_{n}(t),
$$

from formula (10) we can determine (in a non-unique way) - order by order with respect to the perturbative parameter $\lambda$ - the operators $\left\{Z_{n}(t)\right\}_{1}^{\infty}$ and $\left\{C_{n}(t)\right\}_{1}^{\infty}$.

\subsection{Solution corresponding to conditions 1)-3)}

As anticipated, decomposition (6) - or, equivalently, equation (10) - admits infinite solutions. Therefore, it is necessary to impose a gauge condition in order to obtain a specific solution. For instance, as already mentioned, the condition $Z(\lambda ; t)=0, \forall t$, allows to obtain a precise solution, that is the well known Magnus expansion.

In the following, we will use, instead, the gauge fixed by the tern of conditions 1)-3). With these conditions, one can single out a unique solution for the operators $C(\lambda ; t)$ and $Z(\lambda ; t)$; precisely, we get an infinite set of equations that can be solved recursively, order by order, for obtaining the operators $\left\{C_{n}(t)=C_{n}(0)=: C_{n}\right\}_{n=1}^{\infty}$ and $\left\{Z_{n}(t)\right\}_{n=1}^{\infty}$ that appear in the power expansions (11), up to an arbitrary order $N$; specifically, it turns out that

$1^{\text {st }}$ order:

$$
C_{1}=\lim _{\tau \rightarrow \infty} \frac{1}{\tau} \int_{0}^{\tau} \hbar^{-1} \tilde{H}_{1}(t) \mathrm{d} t
$$




$$
Z_{1}(t)=\int_{0}^{t} \hbar^{-1} \tilde{H}_{1}(\mathfrak{t}) \mathrm{d} \mathfrak{t}-C_{1} t-\lim _{\tau \rightarrow \infty} \frac{1}{\tau} \int_{0}^{\tau}\left(\int_{0}^{t}\left(\hbar^{-1} \tilde{H}_{1}(\mathfrak{t})-C_{1}\right) \mathrm{d} \mathfrak{t}\right) \mathrm{d} t
$$

$2^{\text {nd }}$ order:

$$
\begin{aligned}
C_{2}= & \lim _{\tau \rightarrow \infty} \frac{1}{\tau} \int_{0}^{\tau}\left(\frac{i}{2} \operatorname{ad}_{Z_{1}(t)}\left(\hbar^{-1} \tilde{H}_{1}(t)+C_{1}\right)+\hbar^{-1} \tilde{H}_{2}(t)\right) \mathrm{d} t \\
Z_{2}(t)= & \int_{0}^{t}\left(\frac{i}{2} \operatorname{ad}_{Z_{1}(\mathfrak{t})}\left(\hbar^{-1} \tilde{H}_{1}(\mathfrak{t})+C_{1}\right)+\hbar^{-1} \tilde{H}_{2}(\mathfrak{t})\right) \mathrm{d} \mathfrak{t}-C_{2} t \\
& -\lim _{\tau \rightarrow \infty} \frac{1}{\tau} \int_{0}^{\tau}\left(\int_{0}^{t}\left(\frac{i}{2} \operatorname{ad}_{Z_{1}(\mathfrak{t})}\left(\hbar^{-1} \tilde{H}_{1}(\mathfrak{t})+C_{1}\right)+\hbar^{-1} \tilde{H}_{2}(\mathfrak{t})-C_{2}\right) \mathrm{d} \mathfrak{t}\right) \mathrm{d} t
\end{aligned}
$$

Notice that the operators $C_{1}, Z_{1}(t), C_{2}, Z_{2}(t), \ldots$ are indeed selfadjoint, coherently with our previous assumption.

As already mentioned, a typical case occurring in applications (and, in particular, in the application considered in the present paper) is the case where the coefficients of the perturbative expansion of the interaction picture Hamiltonian are operator-valued trigonometric polynomials with respect to the time variable (hence, almost periodic functions of time). In this case, the gauge fixed by conditions 1)-3) is such that the functions $\left\{t \mapsto Z_{n}(t)\right\}_{n=1}^{\infty}$ are zero-mean-valued trigonometric polynomials; as a consequence, all the 'secular terms' are concentrated in the component of the perturbative decomposition of the evolution operator which is generated by the operators $\left\{C_{n}\right\}_{n=1}^{\infty}$, i.e. in the one-parameter group of unitary operators $\{\exp (-i C(\lambda) t)\}_{t \in \mathbb{R}}$.

\subsection{N-th order truncation of the perturbative decomposition (6)}

Once that the operators $C_{1}, Z_{1}(t), \ldots$ have been obtained recursively up to a certain perturbative order $N \geq 1$, one can write the following $N$-th order approximation of the interaction picture evolution operator:

$$
T(\lambda ; t) \approx \exp \left(-i Z^{(N)}(\lambda ; t)\right) \exp \left(-i C^{(N)}(\lambda) t\right) \exp \left(i Z^{(N)}(\lambda)\right),
$$

with

$$
C^{(N)}(\lambda):=\sum_{n=1}^{N} \lambda^{n} C_{n} \quad \text { and } \quad Z^{(N)}(\lambda ; t):=\sum_{n=1}^{N} \lambda^{n} Z_{n}(t) .
$$

We stress that the $N$-th order truncation (14) preserves the fundamental unitary nature of the interaction picture evolution operator $T(\lambda ; t)$.

From formula (14) we find that the overall evolution operator of the system admits the following $N$-th order approximation (recall relation (5)):

$$
\begin{aligned}
U(\lambda ; t) & \approx U_{0}(t) \exp \left(-i Z^{(N)}(\lambda ; t)\right) \exp \left(-i C^{(N)}(\lambda) t\right) \exp \left(i Z^{(N)}(\lambda)\right) \\
& =\exp \left(-i \breve{Z}^{(N)}(\lambda ; t)\right) U_{0}(t) \exp \left(-i C^{(N)}(\lambda) t\right) \exp \left(i Z^{(N)}(\lambda)\right),
\end{aligned}
$$

where $\breve{Z}^{(N)}(\lambda ; t)=U_{0}(t) Z^{(N)}(\lambda ; t) U_{0}(t)^{\dagger}$. From relation (15) it follows that

$$
U(\lambda ; t) \approx \exp \left(-i \breve{Z}^{(N)}(\lambda ; t)\right) U_{\mathrm{eff}}^{(N)}(\lambda ; t) \exp \left(i Z^{(N)}(\lambda)\right),
$$

where $U_{\text {eff }}^{(N)}(\lambda ; t)=U_{0}(t) \exp \left(-i C^{(N)}(\lambda) t\right)$ can be regarded as the evolution operator associated with the effective Hamiltonian

$$
H_{\mathrm{eff}}^{(N)}(\lambda ; t)=H_{0}+\hbar \breve{C}^{(N)}(\lambda ; t) \quad \text { with } \quad \breve{C}^{(N)}(\lambda ; t):=e^{-\frac{i}{\hbar} H_{0} t} C^{(N)}(\lambda) e^{\frac{i}{\hbar} H_{0} t} .
$$


Thus, at the $N$-th perturbative order, $N \geq 1$, the total evolution of the system can be decomposed into a 'dynamical dressing' - i.e. the passage to a generalized interaction picture generated by the time-dependent transformation $\exp \left(-i \breve{Z}^{(N)}(\lambda ; t)\right)$, see [5] - and the evolution generated by an effective Hamiltonian $H_{\text {eff }}^{(N)}(\lambda ; t)$ having the fundamental property that the corresponding interaction picture Hamiltonian, with respect to the reference Hamiltonian $H_{0}-$ namely, $C^{(N)}(\lambda)$ - is time-independent.

\section{Raman schemes: effective coupling}

The perturbative approach described in the preceding section turns out to be a powerful tool for studying Raman schemes and for deducing the 'effective couplings' $|1\rangle \rightleftarrows|2\rangle$. For the sake of definiteness, we consider the situation where two Raman setups are simultaneously present. This case contains that of a single Raman coupling as a special case ${ }^{2}$. Therefore, a direct comparison of the behaviors associated with one or two Raman schemes can be given. It is worth noting that the results obtained for two Raman schemes may be immediately generalized to the case of several Raman couplings. Therefore, the situation under scrutiny allows to illustrate all the relevant conceptually remarkable aspects without introducing any cumbersome notation.

The Schrödinger picture Hamiltonian describing two simultaneously active Raman schemes is given by equations (1) and (2), now taking

$$
\hat{\Omega}_{j 3}(t)=g_{j 3} e^{-i\left(\vec{k}_{j 3} \cdot \vec{r}-\omega_{j 3} t\right)}+g_{j 3}^{\prime} e^{-i\left(\vec{k}_{j 3}^{\prime} \cdot \vec{r}-\omega_{j 3}^{\prime} t\right)}, \quad j=1,2,
$$

where $g_{j 3}, \omega_{j 3}, \vec{k}_{j 3}$, with $j=1,2$, are the coupling constants, frequencies and wave vectors associated with the first couple of Raman lasers, and $g_{j 3}^{\prime}, \omega_{j 3}^{\prime}, \vec{k}_{j 3}^{\prime}$ the analogous quantities for the second Raman scheme. We can assume that $g_{j 3} \neq 0$ (and, of course, $\omega_{j 3} \neq 0, \vec{k}_{j 3} \neq 0$ ), $j=1,2$, so that the special case of a single Raman coupling is recovered for $g_{j 3}^{\prime}=0$. As already mentioned, the operator $\vec{r}$ is the ion center of mass position operator.

In order to generate 'two-photon processes' involving levels $|1\rangle$ and $|2\rangle$ (which is the main feature of the Raman coupling), the ion-laser detunings are fixed in such a way that

$$
\begin{aligned}
& \left(\omega_{3}-\omega_{1}\right)-\omega_{13}=\left(\omega_{3}-\omega_{2}\right)-\omega_{23} \equiv \Delta \neq 0, \\
& \left(\omega_{3}-\omega_{1}\right)-\omega_{13}^{\prime}=\left(\omega_{3}-\omega_{2}\right)-\omega_{23}^{\prime} \equiv \Delta^{\prime} \neq 0 .
\end{aligned}
$$

In the following, we will consider the regime where $\Delta \neq \Delta^{\prime}$; hence, the special case of a single Raman coupling is recovered only for $g_{j 3}^{\prime}=0, j=1,2$. We can also assume, without loss of generality, that $|\Delta| \geq\left|\Delta^{\prime}\right|$, for $g_{j 3}^{\prime} \neq 0$ (and, of course, $\omega_{j 3}^{\prime} \neq 0, \vec{k}_{j 3}^{\prime} \neq 0$ ), $j=1,2$.

We will further suppose that the following high detuning conditions are satisfied:

$$
|\Delta| \gg\left|g_{13}\right|,\left|g_{23}\right|, \nu, \quad(|\Delta| \geq)\left|\Delta^{\prime}\right| \gg\left|g_{13}^{\prime}\right|,\left|g_{23}^{\prime}\right| \text {. }
$$

Passing to the interaction picture with respect to the reference Hamiltonian $H_{0}$, the Schrödinger picture Hamiltonian $H(t)$ is transformed into the interaction picture Hamiltonian

$$
\tilde{H}(t)=H_{\mathrm{B}}+\tilde{H}_{\mathrm{R}}(t)
$$

where

$$
\tilde{H}_{\mathrm{R}}(t)=\left(\hbar \tilde{\Omega}_{13}(t) \hat{\sigma}_{13}+\text { h.c. }\right)+\left(\hbar \tilde{\Omega}_{23}(t) \hat{\sigma}_{23}+\text { h.c. }\right),
$$

\footnotetext{
${ }^{2}$ In Section 2, we have mentioned that the case of a single Raman scheme can be exceptionally treated by a time-independent perturbative method.
} 


$$
\hat{\Omega}_{j 3}(t)=g_{j 3} e^{-i\left(\vec{k}_{j 3} \cdot \vec{r}+\Delta t\right)}+g_{j 3}^{\prime} e^{-i\left(\vec{k}_{j 3}^{\prime} \cdot \vec{r}+\Delta^{\prime} t\right)}, \quad j=1,2 .
$$

At this point, in order to put in evidence the 'natural perturbative parameter' of this model, it will be convenient to introduce the dimensionless (interaction picture) Hamiltonian $\mathfrak{H}(\lambda ; t)$ by setting:

$$
\begin{aligned}
(\hbar \Delta)^{-1} \tilde{H}(t) & =: \mathfrak{H}(\lambda ; t) \\
& =\lambda \sum_{\alpha=x, y, z} \varkappa \hat{a}_{\alpha}^{\dagger} \hat{a}_{\alpha}+\lambda \sum_{j=1,2}\left(\varkappa_{j 3} e^{-i\left(\vec{k}_{j 3} \cdot \vec{r}+\Delta t\right)} \hat{\sigma}_{j 3}+\varkappa_{j 3}^{\prime} e^{-i\left(\vec{k}_{j 3}^{\prime} \cdot \vec{r}+\Delta^{\prime} t\right)} \hat{\sigma}_{j 3}+\text { h.c. }\right),
\end{aligned}
$$

where $\lambda$ is the dimensionless (real) perturbative parameter defined by

$$
\begin{aligned}
& \lambda:=\frac{g}{\Delta}, \quad g \equiv \max \left\{\nu,\left|g_{13}\right|,\left|g_{23}\right|,\left|g_{13}^{\prime}\right|,\left|g_{23}^{\prime}\right|\right\}, \\
& \text { and } \quad \varkappa \equiv \nu / g, \quad \varkappa_{j 3} \equiv g_{j 3} / g, \quad \varkappa_{j 3}^{\prime} \equiv g_{j 3}^{\prime} / g, \quad j=1,2 .
\end{aligned}
$$

We notice explicitly that $0<\varkappa \leq 1,\left|\varkappa_{j 3}\right| \leq 1,\left|\varkappa_{j 3}^{\prime}\right| \leq 1, j=1,2$, and, due to conditions (16), we have that $|\lambda| \ll 1$; hence, $\lambda$ is indeed a 'good perturbative parameter' (compare with the time-independent perturbative approach used in [9] for the single Raman coupling).

Next, applying formulae (12) and (13) to the interaction picture Hamiltonian $\hbar \Delta \mathfrak{H}(\lambda ; t)$, we easily find the following expressions for the operators $C_{1}, C_{2}$ (which, as we have seen, determine the second order effective Hamiltonian):

$$
\begin{aligned}
& \lambda C_{1}=\hbar^{-1} H_{\mathrm{B}}=\nu \sum_{\alpha=x, y, z} \hat{a}_{\alpha}^{\dagger} \hat{a}_{\alpha}, \\
& \lambda^{2} C_{2}=\breve{\omega}_{1} \hat{\sigma}_{11}+\breve{\omega}_{2} \hat{\sigma}_{22}+\breve{\omega}_{3} \hat{\sigma}_{33}+\left(\left(g_{12} e^{-i \vec{k}_{12} \cdot \vec{r}}+g_{12}^{\prime} e^{-i \vec{k}_{12}^{\prime} \cdot \vec{r}}\right) \hat{\sigma}_{12}+\text { h.c. }\right),
\end{aligned}
$$

where we have set

$$
\breve{\omega}_{j}:=-\frac{\left|g_{j 3}\right|^{2}}{\Delta}-\frac{\left|g_{j 3}^{\prime}\right|^{2}}{\Delta^{\prime}}, \quad j=1,2, \quad \breve{\omega}_{3}:=\frac{\left|g_{13}\right|^{2}+\left|g_{23}\right|^{2}}{\Delta}+\frac{\left|g_{13}^{\prime}\right|^{2}+\left|g_{23}^{\prime}\right|^{2}}{\Delta^{\prime}}
$$

and

$$
g_{12}:=\frac{g_{13} g_{32}}{\Delta}, \quad g_{12}^{\prime}:=\frac{g_{13}^{\prime} g_{32}^{\prime}}{\Delta^{\prime}}, \quad \vec{k}_{12}:=\vec{k}_{13}-\vec{k}_{23}, \quad \vec{k}_{12}^{\prime}:=\vec{k}_{13}^{\prime}-\vec{k}_{23}^{\prime},
$$

with $g_{3 j} \equiv g_{j 3}^{*}, g_{3 j}^{\prime} \equiv g_{j 3}^{*}, j=1,2$.

Now, according to what we have shown in Subsection 3.4, the complete temporal evolution of the Raman-driven trapped ion, evaluated within the second order in the perturbative parameter, can be written as

$$
\begin{aligned}
U(\lambda, t) & \approx e^{-\frac{i}{\hbar} H_{0} t} \exp \left(-i Z^{(2)}(\lambda ; t)\right) e^{-i\left(\lambda C_{1}+\lambda^{2} C_{2}\right) t} \exp \left(i Z^{(2)}(\lambda)\right) \\
& =\exp \left(-i \breve{Z}^{(2)}(\lambda ; t)\right) e^{-\frac{i}{\hbar} H_{0} t} e^{-i\left(\lambda C_{1}+\lambda^{2} C_{2}\right) t} \exp \left(i Z^{(2)}(\lambda)\right),
\end{aligned}
$$

where $\breve{Z}^{(2)}(\lambda ; t):=e^{-\frac{i}{\hbar} H_{0} t} Z^{(2)}(\lambda ; t) e^{\frac{i}{\hbar} H_{0} t}$ is an operator-valued function of time, which, as a consequence of of conditions 1$)-3$ ), is a zero-mean-valued trigonometric polynomial, hence contains only 'oscillatory terms'; its explicit form, however, is not relevant for the purposes of the present paper and will be omitted.

Thus, as relation (20) shows, up to the 'dynamical dressing' generated by the time-dependent unitary transformation $\exp (-i \breve{Z}(\lambda ; t)) \approx \exp \left(-i \breve{Z}^{(2)}(\lambda ; t)\right)$, the temporal evolution of the system is described, at the second perturbative order, by the evolution operator $e^{-\frac{i}{\hbar} H_{0} t} e^{-i\left(\lambda C_{1}+\lambda^{2} C_{2}\right) t}$. Such an evolution operator may be regarded as a Schrödinger picture evolutor factorized into the 
the unperturbed evolution $e^{-\frac{i}{\hbar} H_{0} t}$ and the interaction picture evolution $e^{-i\left(\lambda C_{1}+\lambda^{2} C_{2}\right) t}$, which is the one-parameter group of unitary operators generated by the time-independent interaction picture Hamiltonian' $\lambda C_{1}+\lambda^{2} C_{2}$.

The corresponding Schrödinger picture Hamiltonian is then given by

$$
H_{\mathrm{eff}}^{(2)}(t):=H_{0}+e^{-\frac{i}{\hbar} H_{0} t}\left(\lambda C_{1}+\lambda^{2} C_{2}\right) e^{\frac{i}{\hbar} H_{0} t},
$$

and - as it is easily checked using formulae (17) and (18) - it can be decomposed by means of the complementary orthogonal projectors $\hat{P}_{12} \equiv \hat{\sigma}_{11}+\hat{\sigma}_{22}$ and $\hat{\sigma}_{33}\left(\hat{P}_{12}+\hat{\sigma}_{33}=\mathrm{Id},\left[\hat{P}_{12}, \hat{\sigma}_{33}\right]=0\right)$ :

$$
H_{\mathrm{eff}}^{(2)}(t)=H_{\mathrm{eff}}^{(2)}(t) \hat{P}_{12}+H_{\mathrm{eff}}^{(2)}(t) \hat{\sigma}_{33}, \quad\left[H_{\mathrm{eff}}^{(2)}(t), \hat{P}_{12}\right]=\left[H_{\mathrm{eff}}^{(2)}(t), \hat{\sigma}_{33}\right]=0,
$$

where

$$
\begin{aligned}
H_{\mathrm{eff}}^{(2)}(t) \hat{P}_{12}= & \hbar \nu \sum_{\alpha=x, y, z} \hat{a}_{\alpha}^{\dagger} \hat{a}_{\alpha} \hat{P}_{12}+\hbar\left(\omega_{1}+\breve{\omega}_{1}\right) \hat{\sigma}_{11}+\hbar\left(\omega_{2}+\breve{\omega}_{2}\right) \hat{\sigma}_{22} \\
& +\left(\left(\hbar g_{12} e^{-i\left(\vec{k}_{12} \cdot \vec{r}-\omega_{12} t\right)}+\hbar g_{12}^{\prime} e^{-i\left(\vec{k}_{12}^{\prime} \cdot \vec{r}-\omega_{12}^{\prime} t\right)}\right) \hat{\sigma}_{12}+\text { h.c. }\right) \\
H_{\mathrm{eff}}^{(2)}(t) \hat{\sigma}_{33}= & \hbar \nu \sum_{\alpha=x, y, z} \hat{a}_{\alpha}^{\dagger} \hat{a}_{\alpha} \hat{\sigma}_{33}+\hbar\left(\omega_{3}+\breve{\omega}_{3}\right) \hat{\sigma}_{33},
\end{aligned}
$$

with the effective frequencies $\omega_{12}, \omega_{12}^{\prime}$ defined by

$$
\omega_{12}:=\omega_{2}-\omega_{1}=\omega_{13}-\omega_{23}, \quad \omega_{12}^{\prime}:=\omega_{2}^{\prime}-\omega_{1}^{\prime}=\omega_{13}^{\prime}-\omega_{23}^{\prime} .
$$

The result found has a simple and transparent interpretation. In fact, the effective Hamiltonian $H_{\text {eff }}^{(2)}(t)$ is the sum of two completely decoupled Hamiltonians, 'living' respectively in the ranges of the orthogonal projectors $\hat{P}_{12}$ and $\hat{\sigma}_{33}$. It is worth noting the remarkable fact that the Hamiltonian $H_{\mathrm{eff}}^{(2)}(t) \hat{P}_{12}$ can be regarded as the standard Hamiltonian of a trapped two-level ion in interaction with a couple of laser fields characterized, respectively, by the following effective parameters: $g_{12}, \vec{k}_{12}, \omega_{12}$ and $g_{12}^{\prime}, \vec{k}_{12}^{\prime}, \omega_{12}^{\prime}$ (see formulae (19) and (22)).

\section{Conclusions}

We can eventually draw the following conclusions:

- the quantum Hamiltonian describing the physical system of a trapped three-level ion in interaction with a set of laser beams generating Raman couplings can be successfully treated by means of a suitable time-dependent perturbative approach;

- the result of this treatment, in the special case of a single Raman coupling, coincides with the result obtainable by means of a time-independent perturbative approach, see [9];

- at the second perturbative order (hence, with a high degree of accuracy), the dynamics of the system is given, up to a 'dynamical dressing', by the dynamics associated with an effective Hamiltonian describing two decoupled subsystems: an effective laser-driven trapped two-level ion and a simple harmonic oscillator; this result shows also the additivity of the effective couplings: indeed, the effective Hamiltonian associated with a certain set of Raman couplings is characterized by a coupling term which is the sum of the single effective couplings (see formula (21));

- although a detailed analysis of this point is beyond the aim of the present paper, it is worth mentioning the fact that the behavior of certain experimentally observable quantities, in correspondence to certain initial conditions of the system, is rather well reproduced by the effective dynamics only - i.e. neglecting the effect of the dynamical dressing - as 
a consequence of the temporal coarse-graining introduced by the experimental apparatus (which has, unavoidably, a fixed temporal resolution); nevertheless, due to the presence of the dynamical dressing, in addition to the standard Rabi oscillations between the effective levels $|1\rangle$ and $|2\rangle$, also fast transitions coupling these two levels with the auxiliary level $|3\rangle$ take place; then, if the auxiliary level is an excited level with non-negligible decay rate towards (at least one of the) levels $|1\rangle$ and $|2\rangle$, the fast transitions to the auxiliary level, composed with decays, gradually injects decoherence into the effective coherent cycle involving levels $|1\rangle$ and $|2\rangle$ (see [9]), result which is actually in agreement both with experimental observations $[13,14]$ and with numerical simulations on the basis of a phenomenological master equation [15].

A detailed study of the experimentally observable implications of the dynamical dressing, especially with regard to the decoherence effects, is still work in progress [16], and it seems to raise very intriguing issues both on the theoretical and on the experimental side.

\section{Acknowledgements}

The main results of the paper where presented by one of us (B.M.) during the international conference Symmetry in Nonlinear Mathematical Physics (June 20-26, 2005, Kyiv). The authors wish to thank the kind organizers.

[1] Wineland D.J., Monroe C., Itano W.M., Leibfried D., King B.E., Meekhof D.M., Experimental issues in coherent quantum-state manipulation of trapped atomic ions, J. Res. Natl. Inst. Stand. Tech., 1998, V.103, 259-328, quant-ph/9710025.

[2] Leibfried D., Blatt R., Monroe C., Wineland D., Quantum dynamics of single trapped ions, Rev. Modern Phys., 2003, V.75, 281-324.

[3] Ghosh P.K., Ion traps, Oxford, Clarendon Press, 1995.

[4] Steinbach J., Twamley J., Knight P.L., Generation of nonclassical motional states of a trapped atom, Phys. Rev. A, 1997, V.56, 4815-4825.

[5] Aniello P., A new perturbative expansion of the time evolution operator associated with a quantum system, J. Opt. B Quantum Semiclass. Opt., 2005, V.7, S507-S522, quant-ph/0508017.

[6] Aniello P., Man'ko V.I., Marmo G., Porzio A., Solimeno S., Zaccaria F., Trapped ions interacting with laser fields: a perturbative analysis without the rotating wave approximation, J. Russ. Las. Res., 2004, V.25, 30-53, quant-ph/0301138.

[7] Aniello P., Man'ko V., Marmo G., Porzio A., Solimeno S., Zaccaria F., Ion traps in interaction with laser fields: a RWA-free perturbative approach, in Proceedings of the 8-th ICSSUR, Editors H. Moya-Cessa, R. Járengui R., S. Hacyan and O. Castaños, Princeton, Rinton Press, 2003, 17-22.

[8] Aniello P., Perturbative solutions of differential equations in Lie groups, Int. J. Geom. Methods Mod. Phys., 2005, V.2, 111-126.

[9] Militello B., Aniello P., Messina A., Coarse grained and fine dynamics in trapped ion Raman schemes, J. Phys. A: Math. Gen., 2004, V.37, 8177-8187, quant-ph/0310162.

[10] Magnus W., On the exponential solution of differential equations for a linear operator, Comm. Pure Appl. Math., 1954, V.7, 649-673.

[11] Amerio L., Prouse G., Almost periodic functions and functional equations, Van Nostrand Reinhold, 1971.

[12] Wilcox R.M., Exponential operators and parameter differentiation in quantum physics, J. Math. Phys., 1967, V.8, 962-982.

[13] Meekhof D.M., Monroe C., King B.E., Itano W.M., Wineland D.J., Generation of nonclassical motional states of a trapped atom, Phys. Rev. Lett., 1996, V.76, 1796-1799.

[14] Leibfried D., Meekhof D.M., King B.E., Monroe C., Itano W.M., Wineland D.J., Experimental preparation and measurement of the state of motion of a trapped atom, J. Modern Opt., 1997, V.44, 2485-2505.

[15] Di Fidio C., Vogel W., Damped Rabi oscillations of a cold trapped ion, Phys. Rev. A, 2000, V.62, 031802, 4 pages.

[16] Militello B., Aniello P., Messina A., Trapped ion Raman schemes: a novel time-dependent perturbative approach, in preparation. 